\title{
Simulación del campo electromagnético en una línea de transmisión de extra alto voltaje mediante el método de los elementos finitos
}

\author{
Simulation of the electromagnetic field in an extra high \\ voltage transmission line using the finite element \\ method
}

\author{
Krissia. D. Cabezas, Pedro. Jiménez, Juan. D. Ramírez, Raúl. A. Canelos
}

\begin{abstract}
This paper presents the results of the simulation of the electromagnetic field on the San Rafael - El Inga $500 \mathrm{kV}$ overhead transmission line using the Finite Element Method with the FEMM software. The results obtained are contrasted with the measurements made on the site. With AutoCAD, the designs of the structures were raised at 1: 1 scale, which were exported to the FEMM program to carry out the corresponding simulations of the electromagnetic field emitted by the extra high voltage transmission lines. The structures located at different altitudes above sea level were considered and different alternatives of the geometry of the transmission line were simulated to determinate the values of electric and magnetic fields in order to determinate an adequate configuration that presents low values of the electromagnetic field. Finally, through the simulations performed in the FEMM program, the resulting adequate design for an extra-high voltage transmission line is shown.
\end{abstract}

Index Terms - Electromagnetic field, Maxwell's equations, FEMM, overhead transmission line, finite element method

Resumen-En el presente artículo se presentan los resultados de las simulaciones del campo electromagnético en la Línea de Transmisión de 500 kV San Rafael - El Inga mediante el Método de los elementos finitos en el programa FEMM. Los resultados obtenidos son contrastados con las mediciones realizadas con instrumentos de medición en el campo. Con ayuda de AutoCAD se levantaron los diseños de las estructuras a escala 1:1, mismos que fueron exportados al programa FEMM para realizar las simulaciones correspondientes del campo electromagnético emitido por las líneas de transmisión de extra alto voltaje. Se consideraron las estructuras ubicadas en diferentes altitudes sobre el nivel del mar y se simularon diferentes alternativas de la geometría de las torres de la línea de transmisión para determinar los valores del campo eléctrico y magnético hasta obtener una configuración adecuada que presente valores bajos del campo electromagnético. Finalmente, mediante las simulaciones realizadas en el programa FEMM se muestra el diseño adecuado resultante para una línea de transmisión de extra alto voltaje.

Krissia.D. Cabezas, Pedro. Jiménez, Juan. D. Ramírez, and Raúl. A. Canelos are with Departamento de Energía Eléctrica, Escuela Politécnica Nacional, Ecuador, (e-mail: krissia.crubio@outlook.com; pedrodavid.jimenezcorrales@gmail.com; juan.ramirezd@epn.edu.ec; raul.canelos@epn.edu.ec).
Palabras Claves - Campo electromagnético, ecuaciones de Maxwell, FEMM, línea de transmisión, método de elementos finitos

\section{INTRODUCCIÓN}

G $\mathrm{n}$ Ecuador el sistema de transmisión de energía eléctrica al - nivel de $500 \mathrm{kV}$ está compuesto por dos líneas de transmisión en paralelo, de simple circuito, que parten desde la Subestación del proyecto hidroeléctrico Coca Codo Sinclair en el cantón el Chaco de la provincia de Napo y finalizan en la Subestación El Inga en el cantón Quito. La línea de transmisión tiene una longitud total de $126 \mathrm{~km}$. Las rutas de las líneas atraviesan zonas con altitudes que varían entre $1200 \mathrm{~m}$ sobre el nivel del mar (m.s.n.m.) a 4100m.

El transporte de la energía eléctrica puede ocasionar una alteración en el ambiente que genera perturbaciones debido a la presencia del campo eléctrico y magnético, respectivamente. Esta problemática actualmente causa preocupación sobre posibles afectaciones a la salud [1]. Por esta razón se han desarrollado varios estudios e investigaciones para analizar los posibles efectos que produce el campo electromagnético sobre las personas y el ambiente. Actualmente no existen evidencias científicas de los posibles daños que los campos eléctrico y magnético pueden ocasionar, sin embargo, diferentes compañías han preferido realizar técnicas de diseño que disminuyan la emisión de los mismos en las líneas eléctricas [2] con el fin de precautelar que la exposición poblacional y ocupacional a los campos no tenga efectos perjudiciales para la salud. En las líneas de trasmisión de extra alto voltaje, se espera que los valores del campo electromagnético que generan en el medio ambiente estén dentro de los límites establecidos en las normas nacionales e internacionales para campos eléctrico y magnético provenientes de fuentes de baja frecuencia $(60 \mathrm{~Hz})$ [3] [4].

La intensidad del campo eléctrico y magnético se ve influenciada por las condiciones propias de la configuración de la línea (disposición de la geometría de los conductores, distancia de separación entre subconductores, diámetro de cada conductor, separación entre fases, etc.) [5]. 
Las simulaciones se realizan con ayuda del programa FEMM (Finite Element Method Magnetics) para diferentes alternativas geométricas de configuración de las torres que soportan las líneas de transmisión. Estas simulaciones van a permitir obtener valores adecuados de los niveles del campo eléctrico y magnético, de acuerdo a la normativa existente.

Con esos antecedentes, el objetivo de este artículo es utilizar AutoCAD y FEMM para simular el campo electromagnético generado por las líneas de transmisión de 500 kV San Rafael - El Inga y comparar los resultados de las simulaciones con las mediciones realizadas en campo donde se utilizó equipos de medida calibrados que fueron proporcionados por CELEC EP TRANSELECTRIC.

Este artículo presenta en la Sección 2 la metodología aplicada para la simulación del campo electromagnético en la línea de $500 \mathrm{kV}$, la Sección 3 presenta los resultados que se validan con las mediciones realizadas en el campo; para posteriormente observar la influencia de las diferentes condiciones que pueden afectar la intensidad del campo electromagnético medido y simulado. Finalmente, la Sección 4 , resumen las conclusiones y recomendaciones encontradas en el estudio.

\section{Metodología}

En esta sección se detallan las características de los elementos de las estructuras utilizadas para modelar las líneas de transmisión. Se describe el procedimiento a seguir para realizar las diferentes simulaciones de los campos eléctrico y magnético por medio del software FEMM y a su vez se presenta el método aplicado, que, según la norma IEEE Std. 644 se debe seguir para medir el campo electromagnético a lo largo de las líneas de transmisión.

\section{A. Estructuras seleccionadas para el caso de estudio}

Cada una de las fases de la línea de transmisión está constituida por un haz de 4 conductores de aleación de aluminio ACAR 1100 MCM 18/19 y dos cables de guarda, uno de acero y el otro OPGW. Las estructuras metálicas son de tipo "cabeza de gato", configuración horizontal, como se observa en la Fig. 1 [6].

La línea de transmisión está conformada por dos circuitos idénticos llamados A y B. Se escogen tres torres del circuito B ubicadas a diferentes altitudes sobre el nivel del mar: una máxima, una mínima y una media. Se escogió así para el estudio dos torres de retención y una torre de transposición. La Tabla I presenta ciertas características de las estructuras bajo estudio.

TABLA I

CARACTERÍSTICAS DE LAS ESTRUCTURAS BAJO ESTUDIO

\begin{tabular}{ccccccc}
\hline \hline $\begin{array}{c}\text { Torre } \\
\text { No. }\end{array}$ & $\begin{array}{c}\text { Torre } \\
\text { No. AS } \\
\text { BUILT }\end{array}$ & $\begin{array}{c}\text { Altitud }^{\mathbf{1}} \\
(\mathbf{m s n m})\end{array}$ & $\begin{array}{c}\mathbf{A P A}^{\mathbf{2}} \\
\mathbf{( m )}\end{array}$ & $\begin{array}{c}\text { Cross } \\
\mathbf{A R M}^{3} \\
(\mathbf{m})\end{array}$ & $\begin{array}{c}\text { Vano } \\
\text { adelante } \\
(\mathbf{m})\end{array}$ & $\begin{array}{c}\text { Vano } \\
\text { atrás } \\
(\mathbf{m})\end{array}$ \\
\hline GB353N & 229 & 4252.40 & 37.30 & 37 & 308.88 & 690.84 \\
GB378N & 254 & 2976.40 & 24.30 & 24 & 628.94 & 651.04 \\
GB103 & 56 & 1345.64 & 35.30 & 35 & 400.16 & 1078.7 \\
\hline
\end{tabular}

${ }^{1}$ Altura en metros sobre el nivel del mar. ${ }^{2}$ APA: Altura al punto de amarre del conductor. ${ }^{3}$ CROSS ARM: Distancia del mojón a la cruceta.

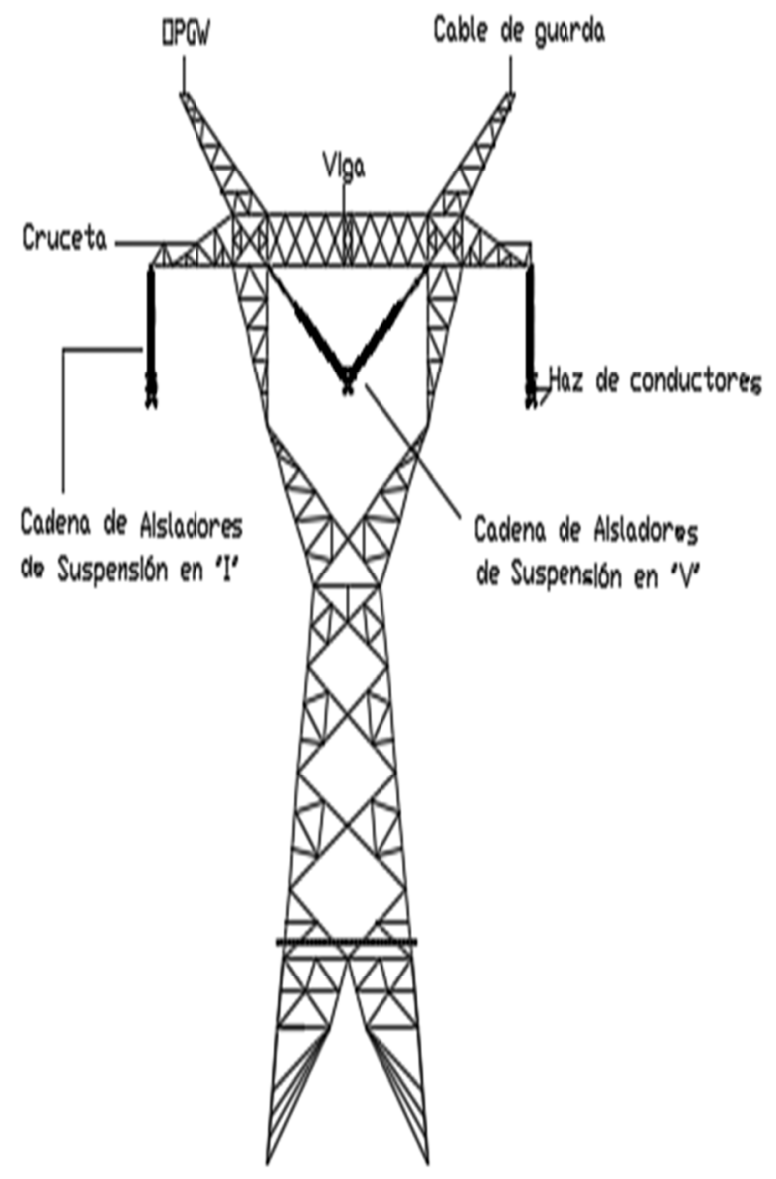

Fig. 1. Estructura de suspensión tipo "cabeza de gato".

En la Fig. 2. se presenta como ejemplo las dimensiones de la estructura 254 de la Tabla I.

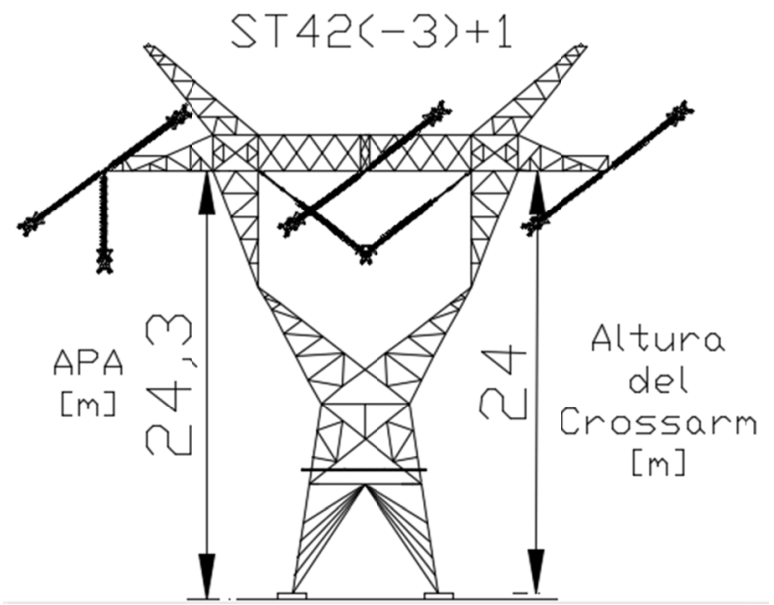

Fig. 2. Vista longitudinal de la estructura 254.

\section{B. Conductores Eléctricos}

El conductor de fase que se utiliza en la línea de transmisión de $500 \mathrm{kV}$ San Rafael - El Inga es el conductor $1100 \mathrm{MCM}$ 18/19 ACAR (Aluminum Conductor Alloy Reinforced). Además, se cuenta con 2 hilos de guarda, uno de acero galvanizado de 7 hilos y $3 / 8$ " de diámetro de alambres de 
acero y otro de fibra óptica denominado OPGW, el mismo que contiene hilos de fibra óptica en su interior y recubiertos por cable de acero galvanizado cuyas especificaciones técnicas se presentan en la Tabla II.

TABLA II CARACTERÍSTICAS DE LAS ESTRUCTURAS BAJO ESTUDIO

\begin{tabular}{ccccc}
\hline \hline Conductor & $\begin{array}{c}\text { Diámetro } \\
(\mathbf{m m})\end{array}$ & $\begin{array}{c}\text { Peso } \\
(\mathbf{k g} / \mathbf{m})\end{array}$ & $\begin{array}{c}\text { Resistencia } \\
(\mathbf{\Omega} / \mathbf{m})\end{array}$ & $\begin{array}{c}\text { Capacidad } \\
\text { mínima de } \\
\text { transporte } \\
(\mathbf{A})\end{array}$ \\
\hline $\begin{array}{c}\text { ACAR 1100 } \\
\text { MCM 18/19 }\end{array}$ & 30.65 & 1.529 & 0.0557 & 474 \\
$\begin{array}{c}\text { Acero } \\
\text { Galvanizado } \\
\text { 3/8" }\end{array}$ & 9.14 & 0.404 & -- & -- \\
$\begin{array}{c}\text { OPGW- } \\
\text { 48B4-90 }\end{array}$ & 13.4 & 0.509 & 0.0557 & -- \\
\hline \hline
\end{tabular}

C. Simulación de los campos eléctrico y magnético en el software FEMM

Para la simulación de los campos eléctrico y magnético en el software FEMM se debe considerar lo siguiente:

- La simulación no es variable en el tiempo.

- El problema se realiza en dos dimensiones en coordenadas cartesianas.

- Los conductores tienen longitud infinita

- La tierra es plana y es un perfecto conductor.

En el modelo inicial se consideran los valores de la Tabla III para los diferentes materiales que componen las estructuras de la línea de transmisión. Sin embargo, estos valores luego de variarán para ajustar el modelo a las mediciones realizadas.

TABLA III

CARACTERÍSTICAS ELECTROMAGNÉTICAS CONSIDERADAS PARA LA SIMULACIÓN

\begin{tabular}{lcccc}
\hline \hline Objeto & Materiales & $\begin{array}{c}\text { Permitividad } \\
\text { eléctrica } \\
\text { Relativa }\end{array}$ & $\begin{array}{c}\text { Permeabilidad } \\
\text { Magnética } \\
\text { Relativa }\end{array}$ & $\begin{array}{c}\text { Conducti } \\
\text { vidad } \\
\text { eléctrica } \\
\text { (MS/m) }\end{array}$ \\
\hline $\begin{array}{l}\text { Aislador } \\
\text { tipo } \\
\text { porcelana }\end{array}$ & Acero & 1000000 & 100 & 1.43 \\
Estructur & Porcelana & $5.9-6.5$ & 10 & $10-100$ \\
a de la & Acero & 1000000 & 10 & $1.00 \mathrm{E}-19$ \\
Torre & Acero & 1000000 & 100 & 1.43 \\
Herrajes & Aluminio & 0 & 100 & 1.43 \\
Aire & Hierro & 0 & $1-1.00032$ & 40.8 \\
& aire & 1.0006 & $1500-20000$ & 11.2 \\
Suelo & semiseco & 3 & 1.00000037 & 2 \\
& Seco & 15 & 12 & $<2.0 \mathrm{E}-7$ \\
& húmedo & 30 & 12 & \\
\hline \hline
\end{tabular}

En el método de elementos finitos, el área en análisis es discretizada en pequeñas regiones finitas conocidas como elementos, generalmente en triángulos o cuadriláteros conectados entre sí por puntos de unión llamados nodos, como se presenta en la Fig. 3. Las incógnitas del problema dejan de ser funciones matemáticas y pasan a ser el valor de estas funciones en los nodos. Mediante la interpolación de los valores conocidos en los nodos se puede llegar a conocer un valor aproximado del modelo analizado. A mayor número de elementos finitos obtenidos, mayor es la exactitud, pero aumenta la complejidad en el planteamiento y resolución del problema [7].



Fig. 3. Torre de transmisión mallada en FEMM.

Una vez discretizado el problema, se obtienen los resultados proporcionados por el programa tanto del campo eléctrico, campo magnético, así como voltaje.

\section{Medición del campo electromagnético en el sitio}

Según la norma IEEE Std. 644-1994 Standard Procedures for Measurement of Power Frequency Electric and Magnetic Fields From AC Power Lines, la medición de intensidad del campo eléctrico y del campo magnético se debe realizar a una altura de 1 metro sobre el nivel del suelo [9].

1) Perfil Longitudinal: La medición debe realizarse a 1 metro sobre el nivel del suelo paralelo a la línea de transmisión, se deben tomar al menos cinco mediciones equidistantes desde la mitad del tramo de la línea de transmisión y en ambas direcciones de tal manera que se cubra toda la longitud del vano [3], [8], como se presenta en la Fig. 4.

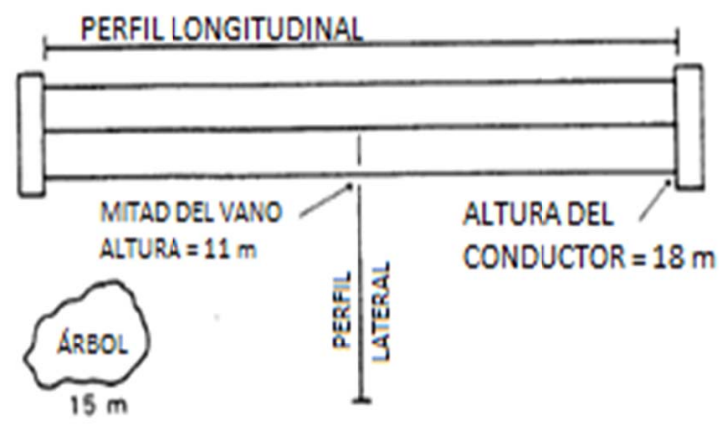

ESCALA: $1 \mathrm{~cm}=50 \mathrm{~m}$

Fig. 4. Medición lateral (transversal) y longitudinal. Vista superior del vano [8].

2) Perfil Lateral: El perfil lateral debe medirse una vez obtenidos los puntos más altos de intensidad del campo eléctrico medidos en el perfil longitudinal. Generalmente la intensidad es mayor en la mitad del vano. Se realiza la 
medición del campo eléctrico y magnético a 1 metro de altura sobre el nivel del suelo en dirección perpendicular a la línea. Las mediciones deben ser realizadas desde el centro de la línea del área de interés y recorrer al menos 30 metros lateralmente desde la ubicación del último conductor, deben tomarse al menos cinco mediciones equidistantes. Es recomendable que se realicen las mediciones lateralmente hasta que se cubra la longitud de la franja de servidumbre [3], [8].

\section{E. Instrumentos de medición}

Existen diferentes instrumentos que permiten medir el campo electromagnético a diferentes frecuencias, en este caso, se consideran instrumentos para medición del campo electromagnético en líneas de transmisión a $60 \mathrm{~Hz}$.

Para el presente estudio se utilizó el instrumento de medida de campos eléctrico y magnético Survey Meter HI-3604. El equipo se presenta en la Fig. 5.

Por otro lado, se utiliza el equipo Hybrid-Powered Environmental Quality Meter 850068 que permite tomar medidas de temperatura y humedad relativa del aire.

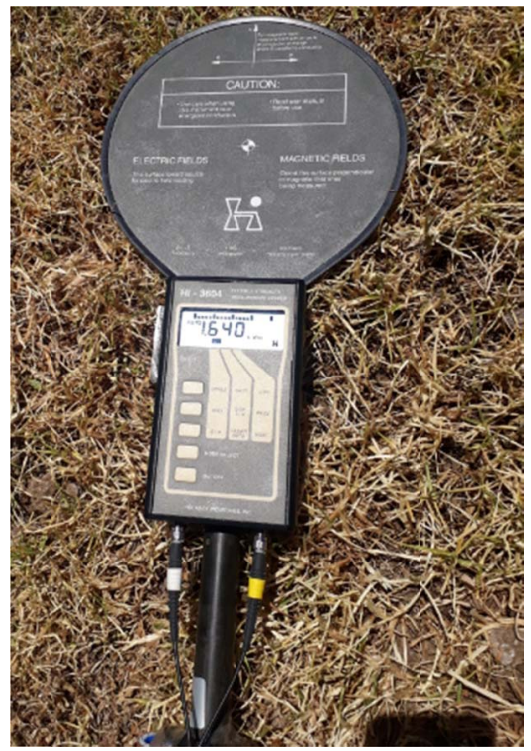

Fig. 5. Survey Meter HI-3604.

\section{F. Procedimiento de simulación}

Se dibujan las 3 torres seleccionadas con sus respectivas estructuras, aisladores, herrajes y conductores a escala real en AutoCAD en formato .dxf, para luego importase al preprocesador de FEMM. En FEMM se escoge el problema electrostático y problema magnético.

Se colocan los parámetros de los materiales que se van a utilizar. En el caso del problema electrostático se requiere tener el valor de la permitividad dieléctrica de los materiales mientras que para el problema magnético se requiere tener el valor de la permeabilidad magnética.

Adicionalmente se definen las condiciones de borde de los conductores según los voltajes de fase más altos del sistema, para ello se considera el voltaje pico que corresponde al voltaje máximo de operación de la línea por fase en estado estable, y la condición de borde de voltaje igual a cero en el suelo. Con la información necesaria para la simulación se realiza la discretización del dominio, donde se generan los triángulos de los elementos finitos en el software.

Finalmente, se obtienen los resultados proporcionados por el programa tanto del campo eléctrico, campo magnético, así como voltaje. Los valores obtenidos pueden ser analizados mediante las gráficas que facilita el software FEMM. En las Fig. 6, Fig. 7 y Fig. 8 se puede apreciar cómo el sofwtare presenta los resultados del campo eléctrico o del magnético. Estos resultados se extraen en un archivo de texto plano .txt para luego ser analizados y presentados en forma de gráficos y de la Tabla del Anexo 1.

\section{RESULTADOS Y DiscusióN}

A continuación, se detallan los resultados obtenidos en la línea de transmisión San Rafael - El Inga. Con los datos obtenidos en la medición se validaron las simulaciones realizadas. Luego se realizaron simulaciones para diferentes casos de estudio y diferentes escenarios.

Los valores medidos deben estar dentro de los límites establecidos en las normas nacionales e internacionales para campos eléctrico y magnético provenientes de fuentes de baja frecuencia $(60 \mathrm{~Hz})$ [3], [4]. En la Tabla IV se presentan los valores límite de exposición para campo eléctrico y magnético a un metro sobre el nivel del suelo.

Para realizar las respectivas simulaciones se consideró el voltaje y corriente que circulaba por la línea de transmisión en el momento que se realizaron las mediciones en el sitio. Se obtuvo el Reporte Post-Operativo Diario del Sistema Nacional de Transmisión para los días en los que se realizó la medición, estos valores se presentan en la Tabla V.

Para la simulación del campo eléctrico y el campo magnético se requieren los parámetros que son calculados a partir de los valores de la Tabla V. Estos parámetros se resumen en la Tabla VI.

TABLA IV

NIVELES DE REFERENCIA PARA LA EXPOSICIÓN A CAMPOS ELÉCTRICO Y MAGNÉTICO DE $60 \mathrm{HZ}$ [3]

\begin{tabular}{cccc}
\hline Tipo de exposición & $\begin{array}{c}\text { Intensidad } \\
\text { del campo } \\
\text { Eléctrico } \\
(\mathbf{E})(\mathbf{V} / \mathbf{m})\end{array}$ & $\begin{array}{c}\text { Intensidad del } \\
\text { campo } \\
\text { Magnético (H) } \\
(\mathbf{A} / \mathbf{m})\end{array}$ & $\begin{array}{c}\text { Densidad } \\
\text { de Flujo } \\
\text { Magnético } \\
(\mathbf{B})(\boldsymbol{\mu T})\end{array}$ \\
\hline $\begin{array}{c}\text { Público en } \\
\text { General } \\
\text { Personal }\end{array}$ & 4167 & 67 & 83 \\
$\begin{array}{c}\text { Ocupacionalmente } \\
\text { expuesto }\end{array}$ & 8333 & 333 & 417 \\
\hline \hline
\end{tabular}

TABLA V

RESUMEN POST-OPERATIVO EN DÍAS DE MEDICIÓN

\begin{tabular}{ccc}
\hline \hline Estructura & $\begin{array}{c}\text { Voltaje } \\
(\mathbf{k V})\end{array}$ & $\begin{array}{c}\text { Potencia } \\
\text { (MVA) }\end{array}$ \\
\hline \multirow{2}{*}{254} & 515.257 & 405.275 \\
229 & 513.291 & 360.906 \\
\multirow{2}{*}{56} & 490.399 & 428.391 \\
& 500.000 & 414.472 \\
\hline \hline
\end{tabular}


TABLA VI

PARÁMETROS PARA SIMULACIÓN DEL CAMPO ELECTROMAGNÉTICO

\begin{tabular}{cccc} 
Estructura & $\begin{array}{c}\text { Voltaje } \\
\text { fase-línea } \\
(\mathbf{k V})\end{array}$ & $\begin{array}{c}\text { Corriente } \\
\text { máxima de un } \\
\text { subconductor en } \\
\text { el haz (A) }\end{array}$ & $\begin{array}{c}\text { Densidad de } \\
\text { corriente } \\
\left.\mathbf{( M A} / \mathbf{m}^{2}\right)\end{array}$ \\
\hline 254 & 420.705 & 160.554 & 0.217604768 \\
229 & 400.409 & 178.314 & 0.241675682 \\
56 & 408.248 & 171.288 & 0.232153423 \\
\hline \hline
\end{tabular}

\section{A. Validación de las simulaciones realizadas}

Con el fin de validar la simulación se redujo la diferencia entre los valores simulados y medidos hasta obtener un error relativo menor al 7\% [10] por medio de la variación de los parámetros de los materiales utilizados. Una vez que no se sobrepasa este error, se pueden tomar los parámetros de la simulación como fijos, con el objetivo de ser utilizados en futuras simulaciones.

La Fig. 6 presenta la distribución del campo eléctrico desde el centro de la torre y se observa que, entre más alta es la torre, el campo eléctrico a nivel del suelo disminuye.

De la misma manera se observa que, no existe presencia del campo eléctrico en el suelo. Las líneas de flujo del campo no penetran la superficie del suelo.

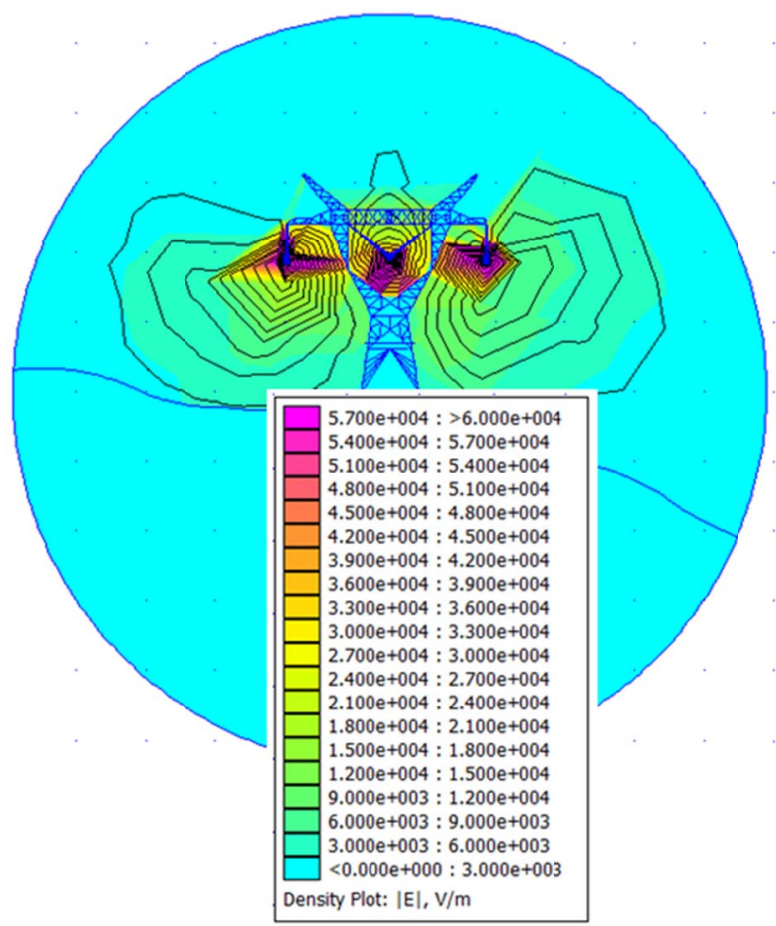

Fig. 6. Distribución del campo eléctrico en una estructura - Vista longitudinal.

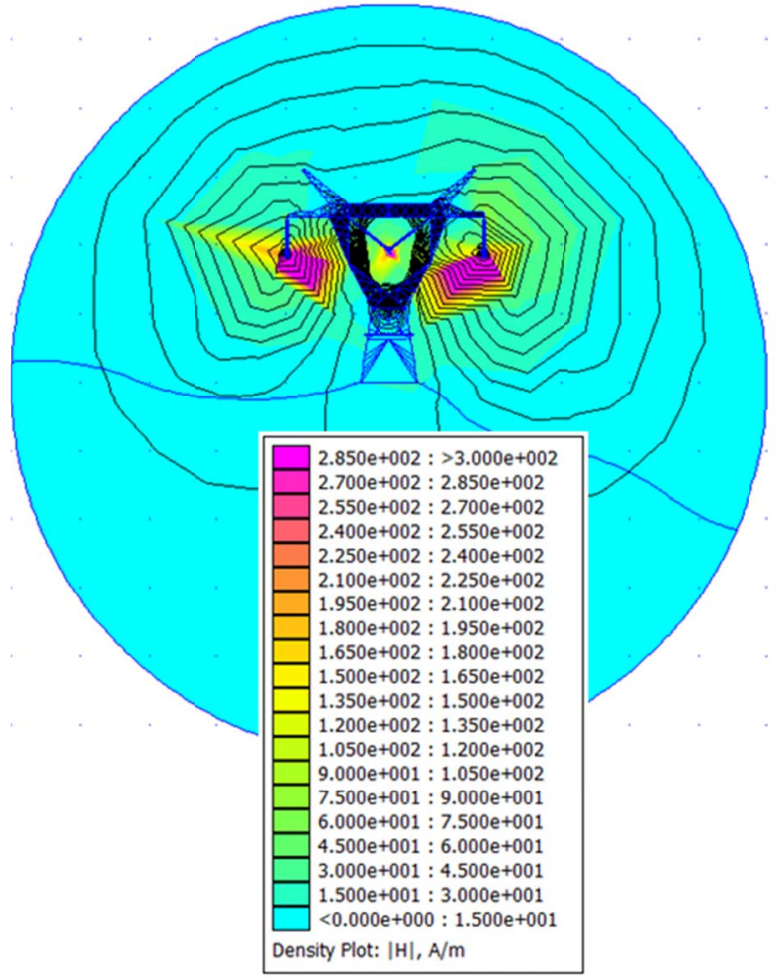

Fig. 7. Distribución del campo magnético en una estructura - Vista longitudinal.

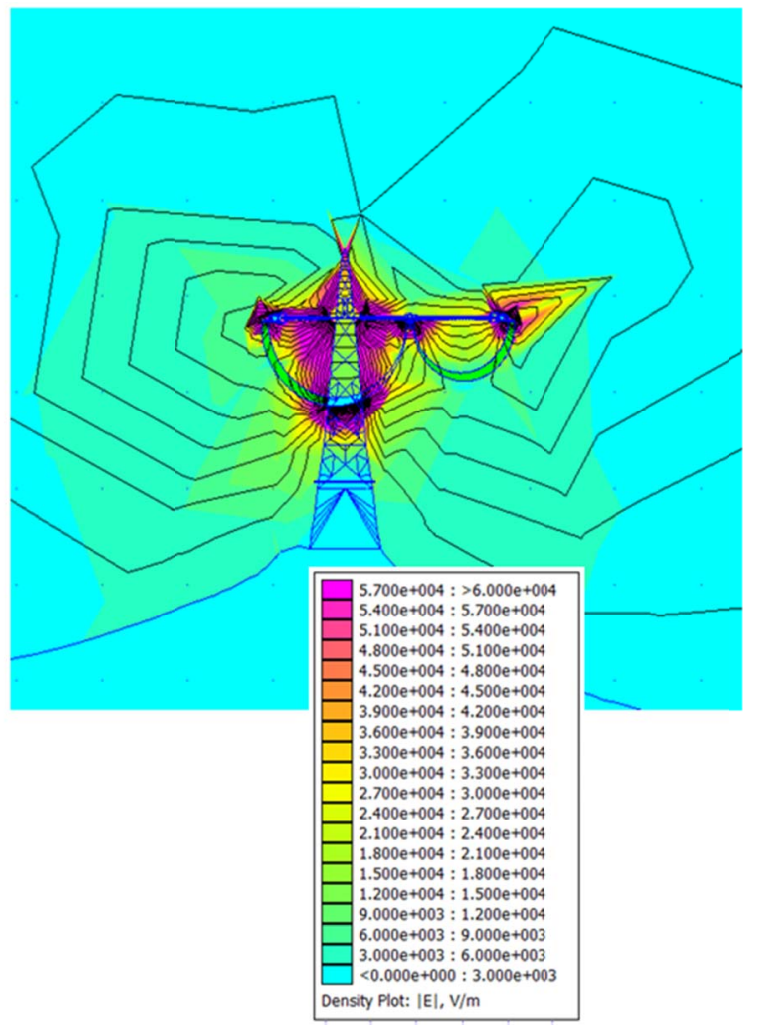

Fig. 8. Distribución del campo eléctrico en una estructura - Vista transversal.

La Fig. 7 presenta la distribución del campo magnético en una estructura. Se observa que existe una mayor presencia del campo magnético cerca del conductor que en la propia 
superficie del suelo. Todo esto relacionado con la cantidad de corriente que circula por el conductor. A diferencia del campo eléctrico, las líneas de flujo penetran fácilmente la superficie del suelo.

La Fig. 8 presenta la distribución del campo eléctrico en una estructura en su vista transversal. Debido a que en el sitio se realizaron las mediciones del campo eléctrico vano adelante (con dirección hacia El Inga) y vano atrás (con dirección hacia San Rafael).

En el Anexo 1 se presenta, como ejemplo, los resultados de la medición del campo electromagnético de la estructura 254 comparados con los resultados de su simulación.

\section{B. Casos de estudio}

Se variaron los parámetros de la configuración geométrica de los conductores, distancia entre fases, número de subconductores por haz, altura de los conductores desde el suelo con el fin de determinar la configuración más adecuada que pueda reducir los valores del campo electromagnético.

Estudios realizados demuestran que la permitividad del aire es inversamente proporcional a la altitud sobre el nivel del mar, mientras que es directamente proporcional a la temperatura y a la presión [9]. Cabe destacar que si bien es cierto que la permitividad del aire puede aumentar o disminuir respecto a la unidad, no lo hace en gran medida.

El valor del campo magnético es directamente proporcional a la corriente de la línea y no sufre perturbación alguna ya sea con presencia de objetos y personas. Para está simulación se considera la corriente máxima por fase de 1894 A. En este caso, se considera la permeabilidad del suelo igual a la unidad 1) Variación de la altura de los conductores sobre el nivel del suelo - Efecto sobre el campo eléctrico



Fig. 9. Efecto de la altura del conductor al suelo a un metro de altura del suelo con respecto al campo eléctrico.

Se considera una permitividad del aire igual a la unidad. En la Fig. 9 se realizan dos simulaciones del campo eléctrico a diferentes alturas de los conductores con respecto al nivel del suelo. La curva de color azul es el resultado del campo eléctrico a una distancia suelo conductor de 18.5 metros, mientras que la curva de color naranja representa el campo eléctrico a una distancia de 31.5 metros. De las gráficas resultantes se observa que, a mayor altura de los conductores, el campo eléctrico a un metro sobre el nivel del suelo disminuye. Para una configuración horizontal, a mayor distancia entre los conductores y el suelo, el campo eléctrico disminuye.

2) Variación de la altura de los conductores sobre el nivel del suelo - Efecto sobre el campo magnético

La Fig. 10, indica que el máximo valor del campo magnético a 18.5 metros (curva azul) sobre el suelo es de $30.193[\mathrm{~A} / \mathrm{m}]$ (37.74 [uT]), pero al aumentar en 1 metro la altura, es decir a 19.5 metros (curva azul), el campo magnético disminuye a $28.695[\mathrm{~A} / \mathrm{m}]$ (35.85 [uT]). En consecuencia, existe una variación de 1.498 [A/m] (1.873 [uT]) al aumentar la altura del conductor al suelo en 1 metro.

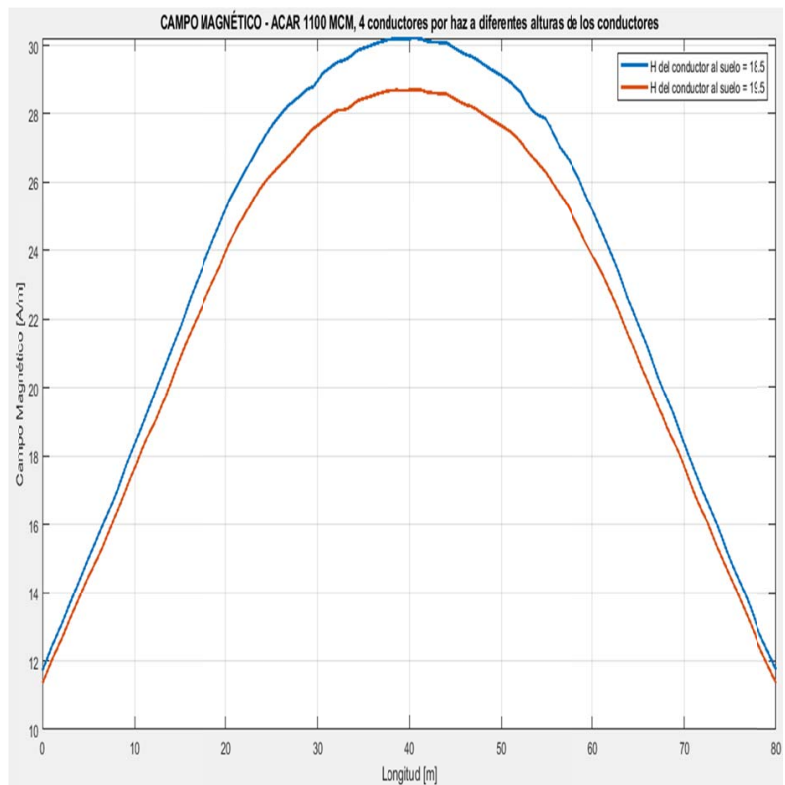

Fig. 10. Efecto de la altura del conductor al suelo a un metro de altura del suelo con respecto al campo magnético.

\section{3) Variación de la de la disposición geométrica de los conductores}

Una reducción significativa del campo electromagnético a lo largo de la línea de transmisión se logra por medio de la selección de una configuración adecuada de la geometría en la estructura de transmisión. Se realizaron simulaciones con diferentes configuraciones como: horizontal, vertical, delta, delta invertido y fase divida. Las geometrías de estas configuraciones se pueden observar con mayor detalle en [11].

También, en base al conductor ACAR 1100 MCM utilizado en la configuración actual, se realiza una comparación de los valores del campo electromagnético al aumentar y disminuir el calibre del conductor a 1000 MCM y 1200 MCM, respectivamente. 


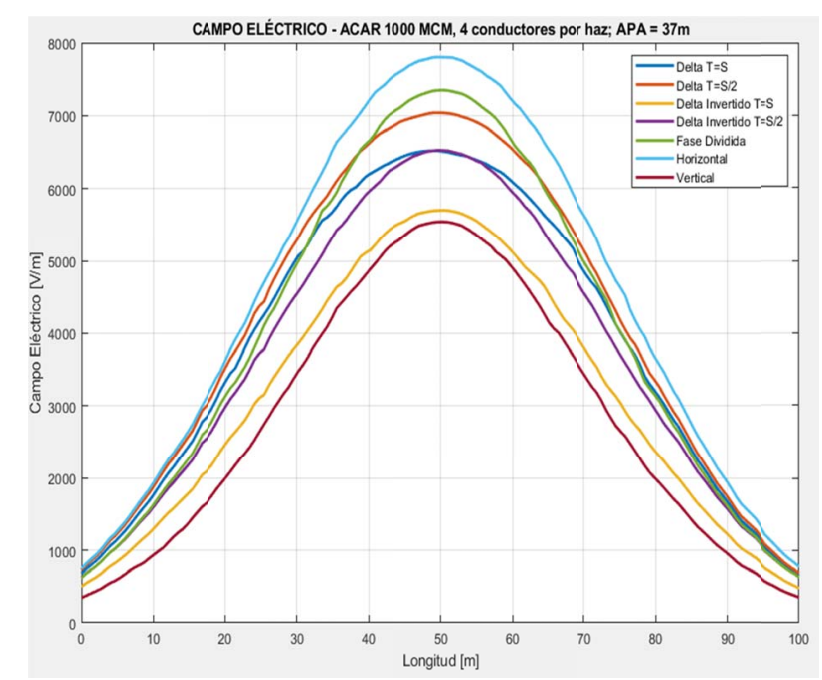

Fig. 11. Campo Eléctrico con diferentes configuraciones de conductores con el conductor ACAR 1000 MCM.

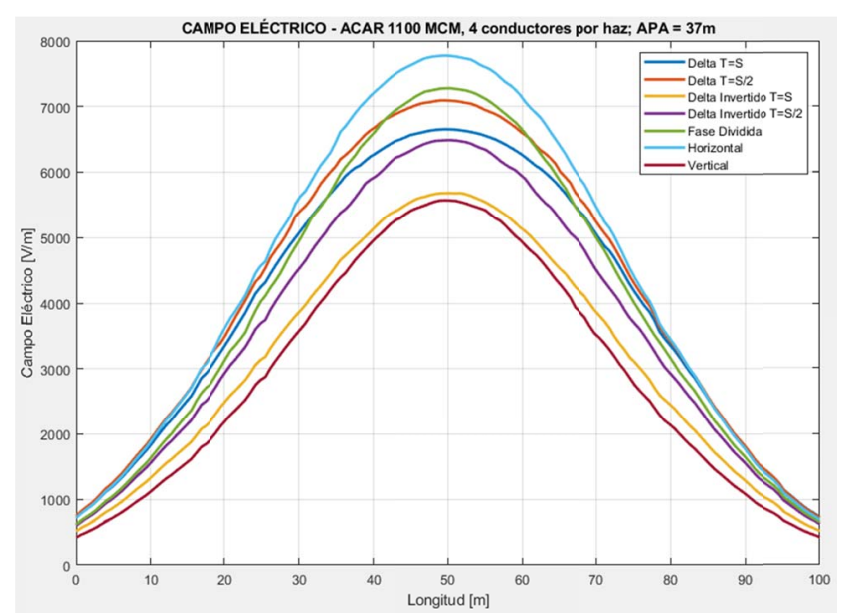

Fig. 12. Campo Eléctrico con diferentes configuraciones de conductores con el conductor ACAR 1100 MCM.



Fig. 13. Campo Eléctrico con diferentes configuraciones de conductores con el conductor ACAR 1200MCM.

De las Fig. 11, Fig. 12 y Fig. 13 se determina que la configuración que reduce considerablemente el valor del campo eléctrico es la configuración vertical.

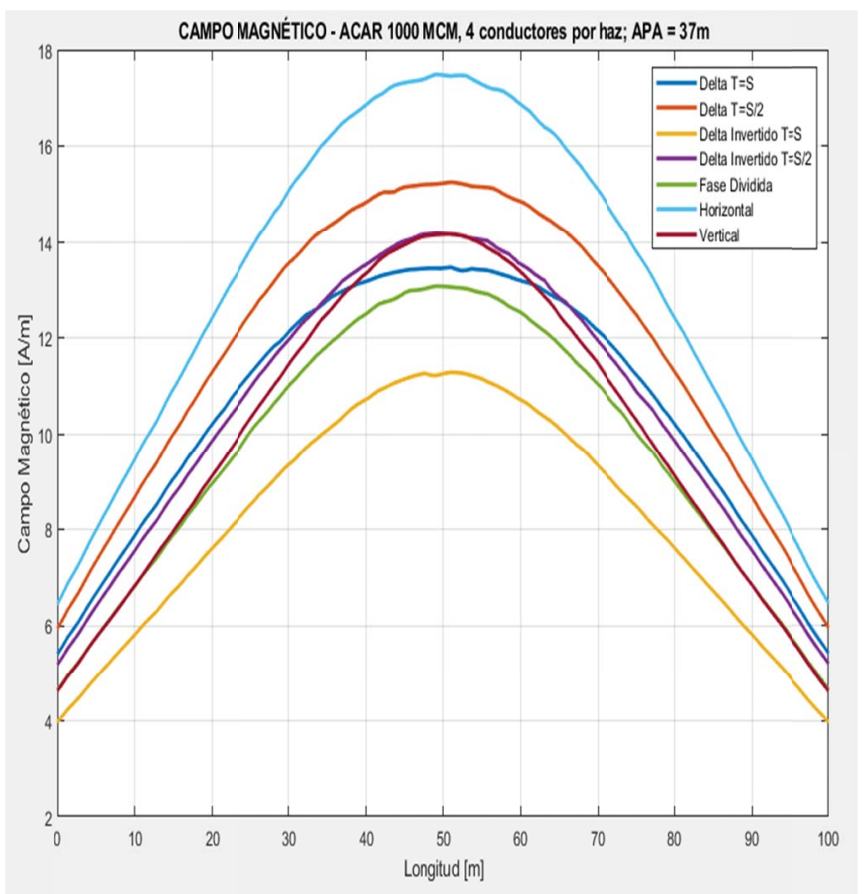

Fig. 14. Campo Magnético con diferentes configuraciones de conductores con el conductor ACAR 1000 MCM.

De las Fig. 14, Fig. 15 y Fig. 16 se determina que la configuración adecuada para reducir el campo magnético es la configuración delta invertido.

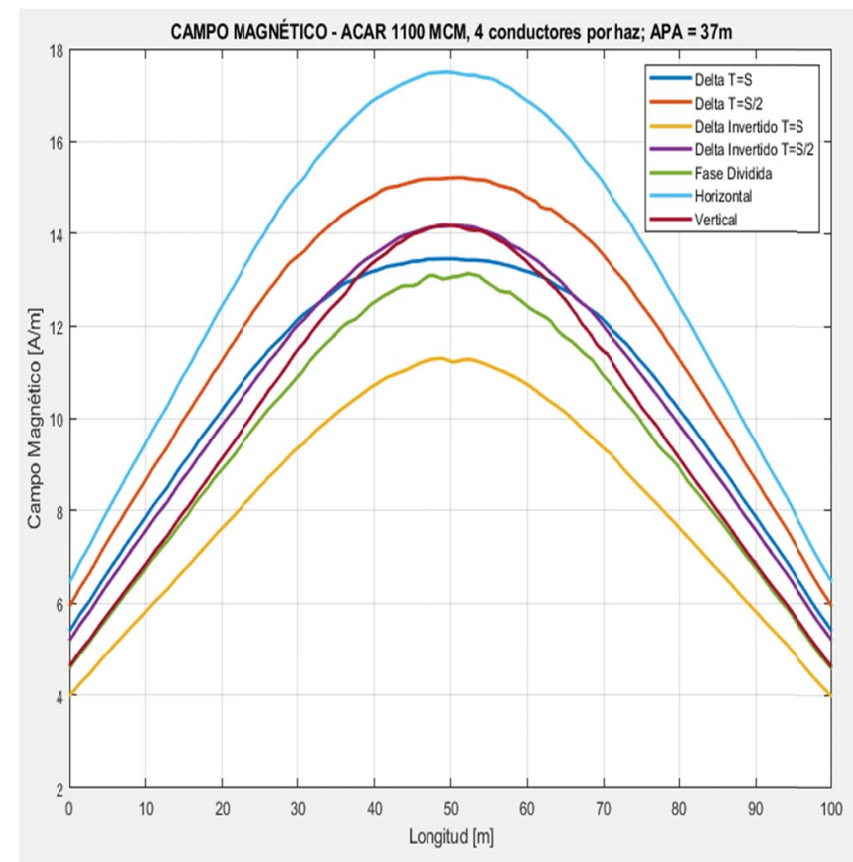

Fig. 15. Campo Magnético con diferentes configuraciones de conductores con el conductor ACAR $1100 \mathrm{MCM}$ 


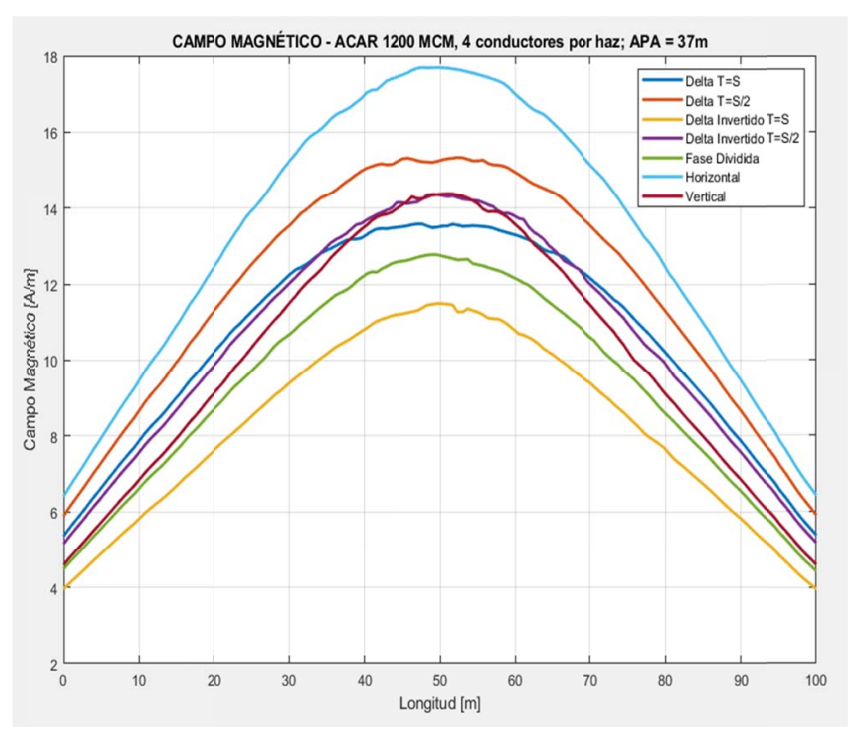

Fig. 16. Campo Magnético con diferentes configuraciones de conductores con el conductor ACAR $1200 \mathrm{MCM}$.

Tanto para el campo eléctrico como el campo magnético, la configuración horizontal es la que produce los valores más altos.

En resumen, la geometría adecuada que podría reducir los valores del campo electromagnético es la configuración vertical. A pesar de que para campo magnético la mejor geometría es delta invertida, este campo depende exclusivamente de la corriente que circula por el conductor, por lo que, en este sentido, las simulaciones del campo eléctrico son las que deciden la geometría adecuada.

Por otro lado, se puede pensar que, al disminuir el valor del campo eléctrico cerca del conductor, el gradiente potencial en la superficie del conductor reduciría, por lo que el efecto corona disminuye y por ende reduzcan las pérdidas asociadas con la formación de este fenómeno.

4) Cambio número de subconductores por haz:

Se han considerado diferentes calibres de conductor según el número " $n$ " de subconductores en el haz a fin de poder observar cómo afecta este parámetro en los valores del campo electromagnético. Se han considerado $\mathrm{n}=3$ conductores de 1700 MCM por haz, $n=4$ conductores de $1100 \mathrm{MCM}$ por haz, y $\mathrm{n}=5$ conductores de $800 \mathrm{MCM}$ por haz.

4.1) Campo eléctrico, configuración horizontal, diferente número de subconductores en el haz, diferentes calibres

En la Fig. 17 se observa el campo eléctrico obtenido bajo el conductor a 1 metro de altura sobre el suelo para diferentes subconductores dentro del haz. Se puede apreciar que, la curva que representa el menor campo eléctrico es la que posee $n=3$ conductores por haz los mismos que tienen un calibre de 1700 MCM. Mientras que para $\mathrm{n}=4$ y $\mathrm{n}=5$, el campo eléctrico es superior.

En efecto, conforme el diámetro del conductor disminuye, el campo eléctrico a nivel del suelo aumenta. Si se aumenta el número de subconductores, el campo eléctrico a nivel del suelo aumenta. Los resultados de algunos puntos de la Fig. 17 se presentan en la Tabla VII, y en la Tabla VIII puede apreciarse la variación porcentual de estos campos eléctricos.

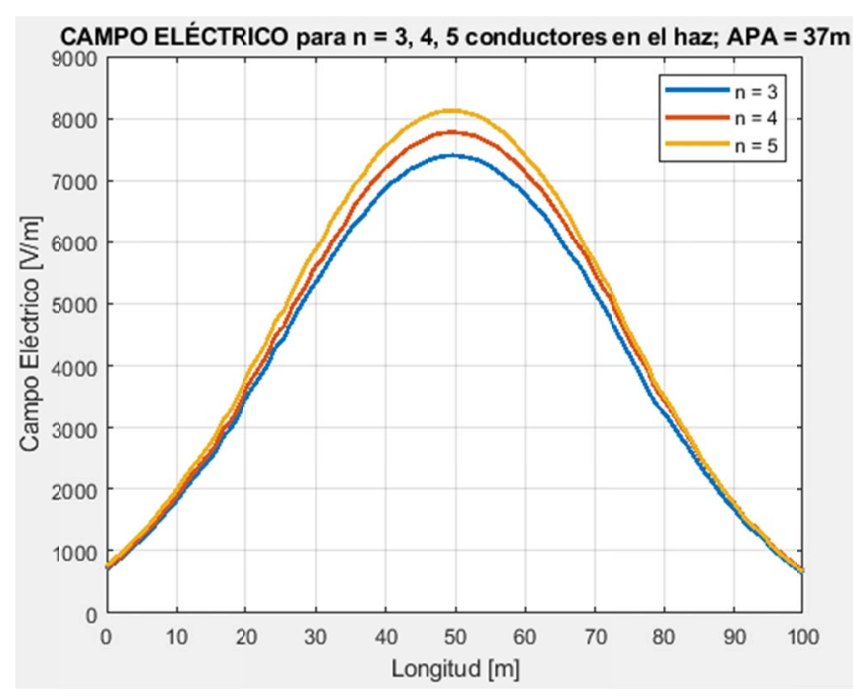

Fig. 17. Campo eléctrico con conductor ACAR $1100 \mathrm{MCM}, \mathrm{n}=4$; ACAR 800 MCM, n = 5; ACAR $1700 \mathrm{MCM}, \mathrm{n}=3$.

TABLA VII

VALORES DEL CAMPO ELÉCTRICO EN (V/M) PARA N=3, N=4, N=5 CONDUCTORES POR HAZ

\begin{tabular}{cccc}
\multicolumn{4}{c}{ CONDUCTORES POR HAZ } \\
\hline \hline Longitud (m) & $\mathbf{n = 3}$ & $\mathbf{n}=\mathbf{4}$ & $\mathbf{n}=\mathbf{5}$ \\
\hline 0 & 696.37 & 716.074 & 758.94 \\
& 4437.58 & 4634.46 & 4878.9 \\
25.50 & & & 9 \\
50.33 & 7397.32 & 7770.89 & 8119.4 \\
& & & 6 \\
75.17 & 4153.28 & 4394.42 & 4714.8 \\
& & & 670.77 \\
\hline
\end{tabular}

TABLA VIII

VARIACIÓN DEL CAMPO ELÉCTRICO CON RELACIÓN A N=4 CONDUCTORES POR

\begin{tabular}{ccc} 
& HAZ & \\
\hline \hline \multirow{2}{*}{ Longitud $(\mathbf{m})$} & $\begin{array}{c}\Delta \mathbf{E}(\%) \\
\mathbf{n}=\mathbf{3}\end{array}$ & $\begin{array}{c}\Delta \mathbf{E}(\%) \\
\mathbf{n}=\mathbf{5}\end{array}$ \\
\hline 0 & -2.75 & 5.99 \\
25.50 & -4.25 & 5.27 \\
50.33 & -4.81 & 4.48 \\
75.17 & -5.49 & 2.74 \\
100.00 & -6.13 & -2.59 \\
\hline \hline
\end{tabular}

\section{2) Campo magnético, configuración horizontal, diferente} número de subconductores en el haz, diferentes calibres

En la Fig. 18 se aprecia el cambio del valor del campo magnético respecto al número de subconductores dentro del haz. En la Fig. 18 se indica que las curvas de color azul y rojo no presentan mayores cambios, mientras que la curva de color amarillo que es la que posee 5 subconductores por haz presenta la mayor brecha en comparación con $n=3$ y $n=4$. Esto quiere decir que, el arreglo de 5 subconductores por haz es el que menor campo magnético presenta a un metro sobre el nivel del suelo. 


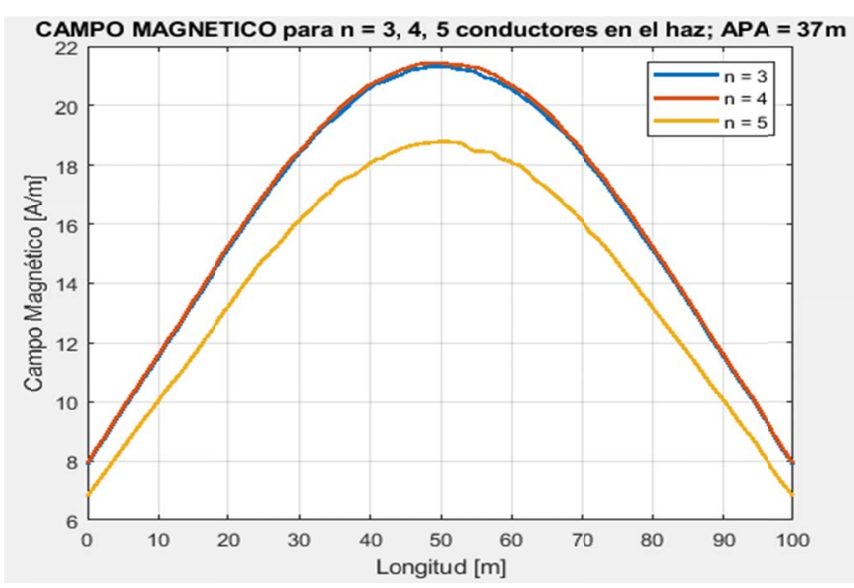

Fig. 18. Campo magnético con conductor ACAR $1100 \mathrm{MCM}, \mathrm{n}=4$; ACAR $800 \mathrm{MCM}, \mathrm{n}=5$; ACAR $1700 \mathrm{MCM}, \mathrm{n}=3$.

Los resultados de algunos puntos de la Fig.18 se presentan en la Tabla IX y en la Tabla X puede apreciarse la variación porcentual de estos campos magnéticos.

El número de subconductores dentro del haz que mejor se ajusta en la reducción del campo electromagnético, para el presente estudio es el haz conformado por 3 subconductores los mismos que tienen un calibre de $1700 \mathrm{MCM}$.

Por lo tanto, la configuración óptima que reduce los valores del campo electromagnético es:

- 3 subconductores por haz

- Configuración vertical $\mathrm{S}=\mathrm{T}$

- Calibre del conductor: 1700 MCM 42/19

La Fig. 19 presenta la dimensión de la estructura adecuada resultante.

TABLA IX

VALORES DEL CAMPO MAGNÉTICO EN (A/M) PARA N=3, N=4, N=5 CONDUCTORES POR HAZ

\begin{tabular}{cccc}
\multicolumn{4}{c}{ CONDUCTORES POR HAZ } \\
\hline \hline Longitud (m) & $\mathbf{n = 3}$ & $\mathbf{n = 4}$ & $\mathbf{n}=\mathbf{5}$ \\
\hline 0 & 7.869 & 7.908 & 6.801 \\
25.50 & 17.029 & 17.117 & 14.903 \\
50.33 & 21.329 & 21.432 & 18.797 \\
75.17 & 16.775 & 16.907 & 14.684 \\
100.00 & 7.869 & 7.908 & 6.810 \\
\hline \hline
\end{tabular}

TABLA X

VARIACIÓN DEL CAMPO MAGNÉTICO O CON RELACIÓN A N=4 CONDUCTORES POR HAZ

\begin{tabular}{ccc}
\hline \hline \multirow{2}{*}{ Longitud (m) } & $\Delta \mathbf{E}(\mathbf{\%})$ & $\Delta \mathbf{E}(\%)$ \\
$\mathbf{n = 3}$ & -0.49 & -13.99 \\
\hline 0 & -0.51 & -12.93 \\
25.50 & -.048 & -12.29 \\
50.33 & -0.78 & -13.14 \\
& -0.49 & -13.88 \\
\hline 75.17 & &
\end{tabular}

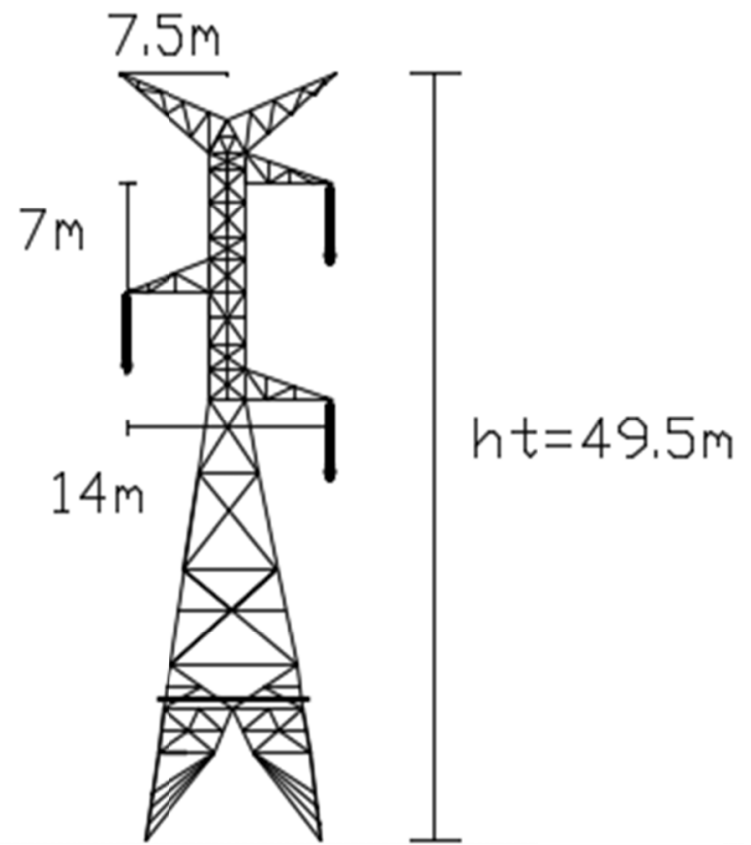

Fig. 19. Configuración vertical, simple circuito.

\section{CONCLUSIONES}

La medición en el sitio permitió contrastar los valores simulados y medidos en este artículo. Se obtuvo una tolerancia de hasta el $7 \%$, como se aprecia en el Anexo 1, en las simulaciones el parámetro ajustable es la permitividad y permeabilidad del aire y suelo que son valores aproximados a la unidad a condiciones estándar de presión y temperatura. Por otro lado, al medir en el lugar donde se encuentran las líneas de transmisión se tienen diferentes temperaturas, presión del aire, densidad del aire, que tienen influencia directa en el campo electromagnético.

Factores como la configuración geométrica de los conductores, la distancia entre fases, el número de subconductores por haz, la altura desde el nivel del suelo al conductor produce una variación en los valores del campo eléctrico y magnético bajo una línea de transmisión. Un adecuado diseño involucra tomar en cuenta estos aspectos de tal manera que los valores de los campos eléctrico y magnético se encuentren dentro de los límites de exposición establecidos en las normas relacionadas.

En las estructuras 56 y 229 los valores de los campos eléctrico y magnético a 1 metro sobre el nivel del suelo se encuentran dentro de los valores establecidos en la norma IEEE Std. 644-1994. Sin embargo, para la estructura 254, en el Anexo 1, el valor medido del campo eléctrico es de 4800 $\mathrm{V} / \mathrm{m}$ el cual excede el valor de campo eléctrico dado por la norma que es de $4167 \mathrm{~V} / \mathrm{m}$. En este caso, dicho valor está fuera de los límites puesto que la altura del punto de amarre APA de los conductores es de 24 metros sobre el nivel del suelo, mientras que el APA de las estructuras 56 y 229 son de 35 y 37 metros, respectivamente. Por otro lado, el campo magnético en las tres estructuras analizadas se encuentra dentro del límite establecido por la norma que es de $67 \mathrm{~A} / \mathrm{m}$.

Las diferentes configuraciones simuladas de la línea de 
transmisión demuestran que, para una reducción en el valor del campo eléctrico y magnético, la disposición geométrica más adecuada es el arreglo de fases de manera vertical. El campo magnético al depender principalmente de la corriente que circula por el conductor no es un factor que decide al momento de seleccionar una configuración adecuada. Lo contrario ocurre con el campo eléctrico que al depender del voltaje de la línea de transmisión puede reducirse según la disposición geométrica de fases utilizada.

Al modificar el número de subconductores del haz en una configuración horizontal con calibre $1100 \mathrm{MCM}$ se tiene que a menor número de subconductores el campo eléctrico es menor, esto se puede apreciar en la Tabla VIII. En este caso, para $\mathrm{n}=3$ el campo eléctrico disminuye en un máximo de $6.13 \%$, mientras que para $\mathrm{n}=5$ el campo eléctrico aumenta hasta en un $5.98 \%$, comparados con $n=4$. En el caso del campo magnético, en las Tablas IX y X se puede observar que para el campo magnético, con 3 subconductores en el haz el campo magnético disminuye muy poco, mientras que para $\mathrm{n}=$ 5 el campo magnético disminuye mucho más. Las variaciones respecto a $n=4$ no sobrepasan el $1 \%$ para el caso de $n=3$, pero llegan hasta un $13.99 \%$ para $n=5$.

\section{REFERENCIAS}

[1] J. Z. Vernieri, P. L. Arnera y M. B. Barbieri, «Aspectos ambientales de campos eléctricos y magnéticos en líneas de alta tensión,» Congreso Internacional de Distribución Eléctrica CIDEL Argentina 2002, p. 7, 2002.

[2] R. J. Caola, D. W. Deno y V. S. W. Dymek, «Measurements of electric and magnetic fields in and around homes near a $500 \mathrm{kV}$ transmission line,» IEEE Transactions on Power Apparatus and Systems, Vols. 1 de 2 PAS-102, nº 10, pp. 3338-3347, Octubre 1983.

[3] R. Oficial, Norma de Radiaciones No Ionizantes de Campos Electromagnéticos, Quito: Editora Nacional, 2007, p. 80.

[4] ICNIRP, «Guidelines for limiting exposure to time-varying electric, magnetic, and electromagnetic fields (Up to $300 \mathrm{GHz}$ ), " pp. 494-515, 1998.

[5] B. Mena, Efecto corona en líneas de transmisión de $500 \mathrm{kV}$, Quito: Escuela Politécnica Nacional, 2008.

[6] C. E. Transeselectric, Estudio de impacto ambiental definitivo. Sistema de transmisión de extra alta tensión y sistemas asociados, Quito, 2013, p. 50 .

[7] D. Meeker, Finite Element Method Magnetics, 4.2 ed., 2015

[8] IEEE Std. 644-1994, «Standard Procedures for Measurement of Power Frequency Electric and Magnetic Fields from AC Power Lines, » p. 31, 1994.

[9] K. A. Dao, V. P. Tran y C. D. Nguyen, «The Wireless Power Transmission Environment from GEO to the Earth and Numerical Estimation of Relative Permittivity vs. the Altitude in the Neutral and Ionized Layers of the Earth Atmosphere,» de International Conference on Advanced Technologies for Communications, 2014.

[10] S. Word, S. Ghania y E. Shaalan, «Three-Dimensional Electric Field Calculation and Measurements inside High Voltage Substations, » p. 4.

[11] Cabezas Rubio, K. D., yJiménez Corrales, P. D. Modelación, simulación y medición de los campos electromagnéticos en una línea de extra alto voltaje mediante el método de elementos finitos, caso de aplicación a línea de transmisión de 500 kV San Rafael-El Inga, Escuela Politécnica Nacional, Quito, 2019. 
ANEXo 1

\begin{tabular}{|c|c|c|c|c|}
\hline \multicolumn{5}{|c|}{ CAMPO ELÉCTRICO } \\
\hline \multicolumn{5}{|c|}{ Perfil Longitudinal } \\
\hline \multicolumn{5}{|c|}{ Vano adelante } \\
\hline \multirow{2}{*}{$\begin{array}{l}\text { Ubicación del punto } \\
\text { de medición }\end{array}$} & Distancia recorrida del vano [m] & \multicolumn{2}{|c|}{$\mathrm{E}[\mathrm{kV} / \mathrm{m}]$ a 1 metro de altura } & \multirow{2}{*}{ \%Error } \\
\hline & & Medido & Simulado & \\
\hline 0 & Dentro de la torre & 0.1731 & 0.180358 & 4.19 \\
\hline 1 & 5 & 3.7146 & 3.84 & 3.29 \\
\hline 2 & 10 & 3.58535 & 3.79 & 5.66 \\
\hline 3 & 15 & 2.92425 & 2.85 & 2.42 \\
\hline 4 & 20 & 2.72626 & 2.80 & 2.58 \\
\hline 5 & 25 & 2.67547 & 2.81 & 5.10 \\
\hline 6 & 30 & 2.13762 & 2.09 & 2.06 \\
\hline 7 & 35 & 1.98801 & 2.12 & 6.53 \\
\hline 8 & 40 & 1.95445 & 2.06 & 5.59 \\
\hline 9 & 45 & 1.35278 & 1.44 & 6.63 \\
\hline 10 & 50 & 1.3506 & 1.41 & 4.11 \\
\hline 11 & 60 & 0.93152 & 0.92 & 1.53 \\
\hline 12 & 70 & 0.55821 & 0.57 & 2.44 \\
\hline 13 & 80 & 0.49477 & 0.51 & 3.52 \\
\hline 14 & 90 & 0.28832 & 0.277848 & 3.63 \\
\hline 15 & 100 & 0.35263 & 0.373109 & 5.81 \\
\hline 16 & 110 & 0.21973 & 0.21 & 5.77 \\
\hline 17 & 120 & 0.19725 & 0.20 & 0.28 \\
\hline 18 & 130 & 0.15942 & 0.17 & 5.45 \\
\hline 19 & 140 & 0.10136 & 0.10 & 5.38 \\
\hline
\end{tabular}

Ubicación del punto de medición
Distancia recorrida del vano $[\mathrm{m}]$

$\mathrm{E}[\mathrm{kV} / \mathrm{m}]$ a 1 metro de altura

\%Error

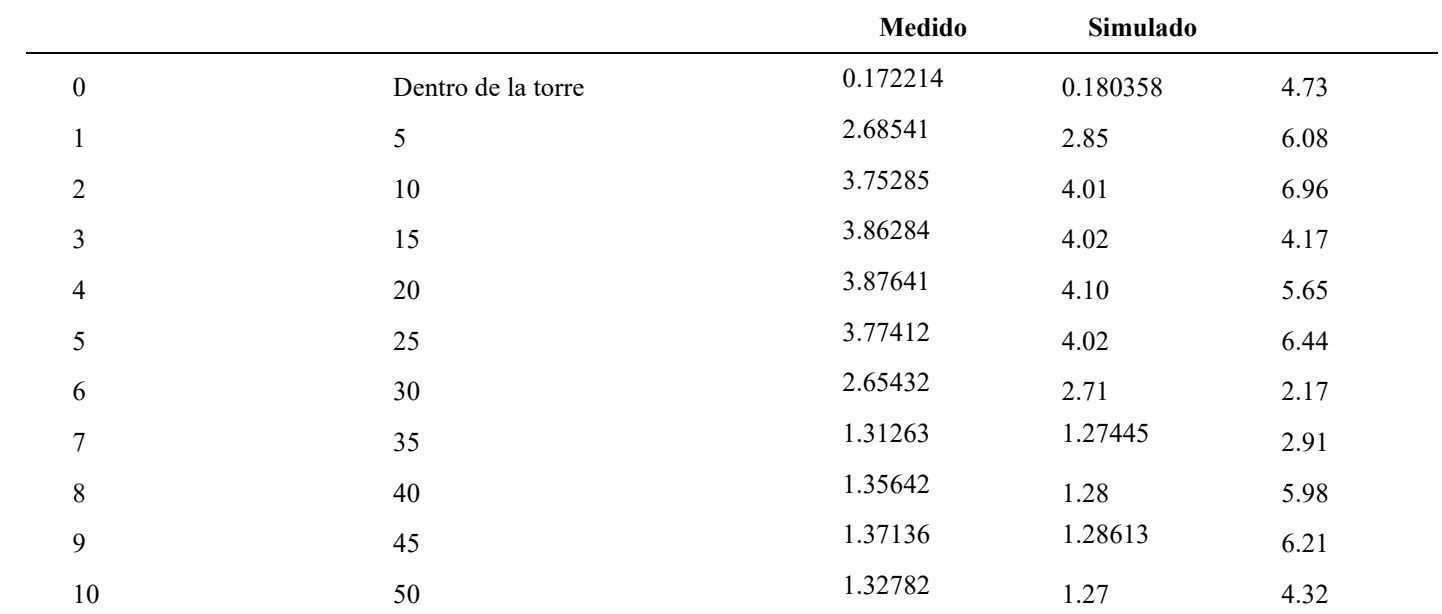




\begin{tabular}{lllll}
11 & 60 & 1.0221 & 0.96 & 6.50 \\
12 & 70 & 0.43623 & 0.41 & 6.35 \\
13 & 80 & 0.38928 & 0.41 & 6.56 \\
14 & 90 & 0.36425 & 0.39 & 6.38 \\
15 & 100 & 0.25478 & 0.24 & 4.96 \\
16 & 110 & 0.23621 & 0.25 & 3.88 \\
17 & 120 & 0.14838 & 0.16 & 4.96 \\
18 & 130 & 0.11025 & 0.11 & 2.85 \\
19 & 140 & 0.10124 & 0.11 & 6.78 \\
20 & 150 & 0.09615 & 0.10 & 3.90 \\
\hline
\end{tabular}

Lado izquierdo Campo Eléctrico

\begin{tabular}{|c|c|c|c|c|}
\hline \multirow{2}{*}{$\begin{array}{l}\text { Ubicación del punto } \\
\text { de medición }\end{array}$} & \multirow{2}{*}{ Distancia recorrida del vano [m] } & \multicolumn{2}{|c|}{$\mathrm{E}[\mathrm{kV} / \mathrm{m}]$ a 1 metro de altura } & \multirow{2}{*}{ \%Error } \\
\hline & & Medido & Simulado & \\
\hline 1 & 10 & 4.69 & 4.439 & 5.35 \\
\hline 2 & 20 & 4.8 & 4.891 & 1.90 \\
\hline 3 & 30 & 2.79 & 2.848 & 2.08 \\
\hline \multicolumn{5}{|c|}{ Lado derecho Campo Eléctrico } \\
\hline \multirow{2}{*}{$\begin{array}{l}\text { Ubicación del punto } \\
\text { de medición }\end{array}$} & \multirow[t]{2}{*}{ Distancia recorrida del vano [m] } & \multicolumn{2}{|c|}{$\mathrm{E}[\mathrm{kV} / \mathrm{m}]$ a 1 metro de altura } & $\%$ Error \\
\hline & & Medido & Simulado & \\
\hline 1 & 10 & 3.812 & 4.069 & 6.74 \\
\hline 2 & 20 & 2.321 & 2.251 & 3.02 \\
\hline 3 & 30 & 1.71 & 1.605 & 6.14 \\
\hline \multicolumn{5}{|c|}{ Lado izquierdo Campo Magnético } \\
\hline \multirow{2}{*}{$\begin{array}{l}\text { Ubicación del punto } \\
\text { de medición }\end{array}$} & \multirow{2}{*}{ Distancia recorrida del vano [m] } & \multicolumn{2}{|c|}{ B [uT] a 1 metro de altura } & $\%$ Error \\
\hline & & Medido & Simulado & \\
\hline 1 & 10 & 9.5725 & 9.91 & 3.50 \\
\hline 2 & 20 & 7.65125 & 7.94 & 3.78 \\
\hline 3 & 30 & 6.655 & 6.82 & 2.42 \\
\hline \multicolumn{5}{|c|}{ Lado derecho Campo Magnético } \\
\hline \multirow{2}{*}{$\begin{array}{l}\text { Ubicación del punto } \\
\text { de medición }\end{array}$} & \multirow[t]{2}{*}{ Distancia recorrida del vano $[\mathrm{m}]$} & \multicolumn{2}{|c|}{ B [uT] a 1 metro de altura } & $\%$ Error \\
\hline & & Medido & Simulado & \\
\hline 1 & 10 & 11.12 & 11.80 & 6.12 \\
\hline 2 & 20 & 7.15625 & 7.38 & 3.07 \\
\hline 3 & 30 & 5.3425 & 5.63 & 5.45 \\
\hline
\end{tabular}

